\title{
Simple Method for Measuring Small Retardance
}

\author{
N. N. Nagib ${ }^{1 *}$--- A. W. Abdallah ${ }^{2}$-- M. S. Bahrawi ${ }^{3}$--- H. Osman ${ }^{4}$--- N. A. Mahmoud ${ }^{5}$--- E. \\ S. Mousa ${ }^{6}$
}

${ }^{i^{*}, 2,9,5}$ National Institute for Standards, Giza, Egypt.

Email:nabil_nagib@yahoo.com

${ }^{4,6}$ Physics Department, Faculty of Science, Cairo University, Giza, Egypt.

\begin{abstract}
Small retardances are encountered in many experimental works. Internal stresses, weakly birefringent materials, optical windows and formation of contaminating surface layers are sources of small retardances. Most known methods for retardance measurements fail to determine accurately their values which are sometimes essential in the evaluation of experimental results. In this work, we present a method for accurate measurement of a small retardance. Our study aims to find the retardance error in a birefringent full-wave plate which, if perfect, is considered as of zero retardance. Our treatment will make use of a previously presented model for simultaneous calibration of two phase plates.
\end{abstract}

Keywords: Retardance measurement, Small retardance, Accuracy of measurement.

Licensed: This work is licensed under a Creative Commons Attribution 4.0 License.

\section{Introduction}

It was shown in a previous work [1] that two phase plates $C 1$ and $C 2$ could be calibrated simultaneously at any wavelength using the optical arrangement $P-C 1-C 2-A$, Figure 1, where $P$ and $A$ are polarizing prisms and $C 1$ and $C 2$ are oriented at $c 1=45^{\circ}$ and $c 2=O^{\circ}$ (small letters $p, c 1, c 2$ and $a$ are used for the orientations of the elements and angles are measured by an observer receiving the radiation in a CCW sense from the positive $\mathrm{x}$-direction with respect to the transmission axes of the polarizers and the fast axes of the plates). By adjusting $P$ and $A$ for extinction, two extinction pairs $(p 1, a 1)$ and $(p 2, a 2)$ could be obtained such that

$$
\begin{aligned}
& p^{2}=p_{1} \pm 90^{\circ}, \\
& a 2=a 1 \pm 90^{\circ} .
\end{aligned}
$$

The retardances $\delta 1$ and $\delta 2$ of $C 1$ and $C 2$ are then given by the simple expressions

$$
\begin{aligned}
& \cos \delta 1=-\cos 2 a / \cos 2 p, \\
& \cos \delta 2=-\sin 2 p / \sin 2 a .
\end{aligned}
$$

where $p, a$ refer to any of the two extinction pairs.

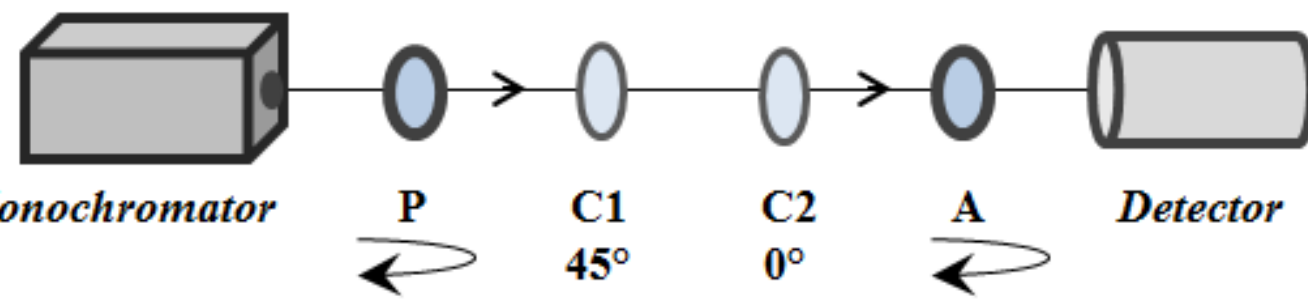

Figure-1. The optical system for simultaneous calibration of two phase plates. $\mathrm{P}$ and $\mathrm{A}$ - polarizing prisms, $\mathrm{C} 1$ and $\mathrm{C}_{2}-$ the calibrated plates and $\mathrm{D}$ is the detecting system. 
This model represents a special calibration case in the sense that it does not employ a standard reference. Here each of the two calibrated plates acts as reference for the other plate. In using this model for the measurement of small retardances, serious errors occur in the retardances of the plates corresponding to small errors in the extinction values of $p$ or $a$. Thus, consider two plates $C 1$ and $C 2$ of retardances $\delta 1=3^{\circ}$ and $\delta 2=$ $6^{\circ}$. It follows from Equations $2 \mathrm{a}, \mathrm{b}$ that one of the extinction pairs is $(p, a)=(13.2355,-76.6860)$. This pair gives the correct values of $\delta 1$ and $\delta 2$. Consider now an error of $0.1^{\circ}$ in the reading of $P$ such that the extinction pair is read as $(13.1355,-76.6860)$. Applying Equations 2a,b we get $\delta 1=4.51^{\circ}$ and $\delta 2=9.05^{\circ}$. This shows that an error of $0.1^{\circ}$ in the reading of $P$ results in corresponding errors of $50 \%$ in the retardances of the plates. On the other hand, if the same error of $0.1^{\circ}$ occurs in the reading of $A$ such that $(p, a)=(13.2355,-76.7860)$, no results will be obtained as both values of $\cos \delta 1$ and $\cos \delta 2$ will exceed unity.

In a previous study [2], it was shown that if the retardance $\delta 1$ (of $C 1$ ) in our system varies from $O^{\circ}$ to $180^{\circ}$ while $\delta 2$ is of fixed quarterwave retardance, then the error in the measured value of $\delta 2$ corresponding to a small error in the reading of $P$ drastically increases for values of $\delta 1$ close to $O^{\circ}$ or $180^{\circ}$, Figure 3 in Ref. [2]. For values of $\delta 1$ close to $90^{\circ}$ (between $70^{\circ}$ and $110^{\circ}$ ), the error in $\delta 2$ is $\sim 2 \Delta p$ where $\Delta p$ is the error in the reading of $P$. It was concluded that the calibration system is weakly sensitive to errors (type $A$ or type $B$ ) in the settings of the elements if the calibrated plates have retardances close to $90^{\circ}$.

\section{Measurement of Small Retardance}

If a plate $C$ of small retardance is introduced in the system $P-C 1-C 2-A$ discussed above between two plates $C 1$ and $C 2$ of moderate retardance values $\left(70^{\circ}-110^{\circ}\right)$, then its retardance $\delta$ will be added to (or subtracted from) $\delta 1$ if its fast axis is oriented at $45^{\circ}$ or $-45^{\circ}$, In this case, Eqs. 2 (a, b) change to
$\cos (\delta 1 \pm \delta)=-\cos 2 a / \cos 2 p$
$\cos \delta 2=-\sin 2 p / \sin 2 a$.

The positive or negative sign in $\left(3\right.$, a) corresponds to $C$ oriented at $45^{\circ}$ or $-45^{\circ}$. On the other hand, if $C$ is oriented at $0^{\circ}\left(\right.$ or $\left.90^{\circ}\right)$, we have

$$
\begin{aligned}
& \cos \delta 1=-\cos 2 a / \cos 2 p, \\
& \cos (\delta 2 \pm \delta)=-\sin 2 p / \sin 2 a .
\end{aligned}
$$

Again, the positive (negative) sign is used if $C$ and $C 2$ have similar (orthogonal) orientations.

\section{Experimental}

Three quartz phase plates were used in the experimental work. The first two plates $C_{t}$ and $\mathrm{C}_{2}$ are of nominal quarterwave retardance at $632.8 \mathrm{~nm}$. The two plates were previously calibrated using an accurate photometric method [3] and found to have retardances of $\delta_{1}=91.66^{\circ}$ and $\delta_{2}=88.33^{\circ}$. The third plate $C$ is a composite plate consisting of two half-wave plates each of nominal value $\lambda / 2$ at $632.8 \mathrm{~nm}$ aligned with their fast axes parallel to provide a full-wave plate. The plate $C$ is then treated as a single full-wave plate. Note that an exact full-wave plate is equivalent to a plate of zero retardance since retardance is measured between $O^{\circ}$ and $360^{\circ}$. The plate $C$ was placed with the fast axis set at $45^{\circ}$ between two polarizing prisms set at $0^{\circ}$ and $90^{\circ}$, Figure 2 and the wavelength of the incident beam is changed around the value $632.8 \mathrm{~nm}$ until the emergent beam is extinguished. The wavelength value at extinction was found to be $634.8 \mathrm{~nm}$ At this wavelength the plate is of exact $2 \pi$ retardance (or equivalently of zero retardance). It is then expected that the composite plate $C$ will have a small retardance $\delta$ at $632.8 \mathrm{~nm}$ since the retardance increases at smaller wavelength.

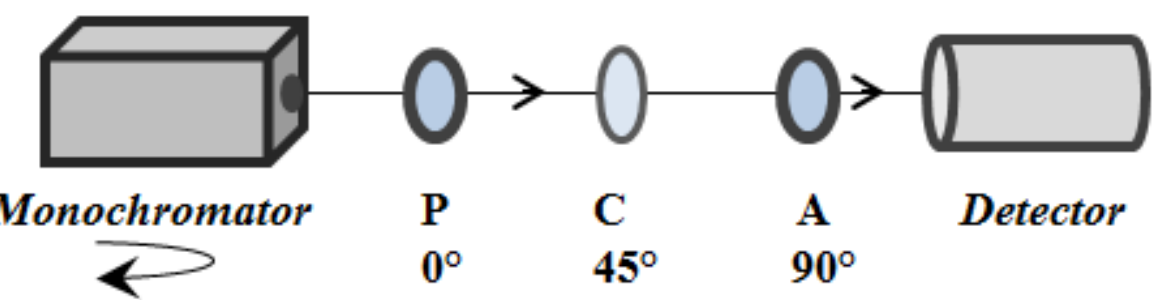

Figure-2. Calibration of the complex full-wave plate.

In the experimental work at $632.8 \mathrm{~nm}$, four arrangements were considered. The first two arrangements, Figure 3.a were with $C$ set between $C_{1}$ and $C_{2}$ with its fast axis at $45^{\circ}$ or at $-45^{\circ}$. Two extinction pairs were recorded for each of the two arrangements. The other two cases were with $C$ oriented at $0^{\circ}$ or $90^{\circ}$ and again four results were recorded, Figure 3.b It was found that

$$
\delta=(1.26 \pm 0.08)^{\circ}
$$

where 0.08 is the standard deviation of the mean. 


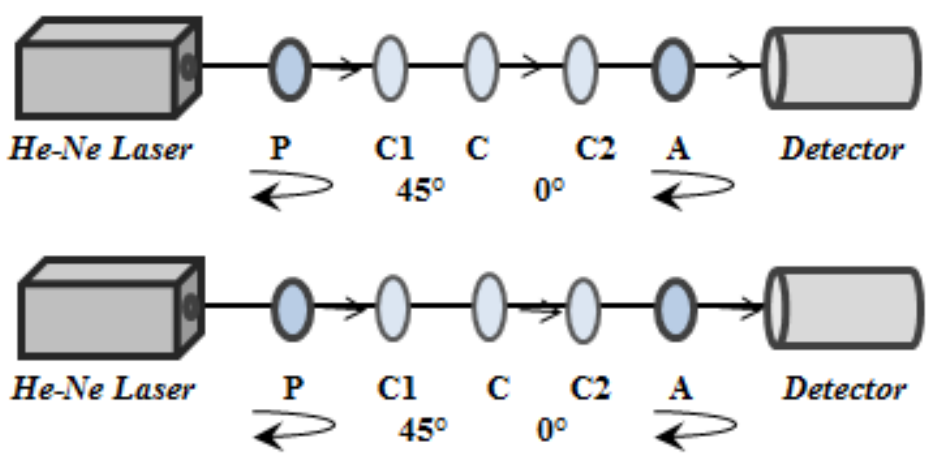

Figure-3(a,b). The optical system for calibrating a place of small retardance C. Symbols are the same as in Figure 1 and $\mathrm{C}$ is the calibrated complex full-wave plate of small retardance at $632.8 \mathrm{~nm}$.

\section{Discussion}

We showed first that the model $P-C 1-C 2-A$ fails to provide accurate results if one (or both) of the measured plates is of small retardance. It might be thought that other techniques provide accurate results for small retardance measurement. Considering the Senarmont compensator method [4], we find that the inaccuracy in measuring small retardances still persists. In the Senarmont method, the same arrangement $P$ $C 1-C 2-A$ is used with $P$ oriented at $O^{\circ}$ and $C 2$ is of exact quarterwave retardance at the working wavelength. $C 1$ and $C 2$ are oriented at $45^{\circ}$ and $O^{\circ}$ just as in our system. The analyzer $A$ is rotated for extinction and the retardance $\delta 1$ of $C l$ is given from the relation

$$
\delta 1=2 a+\pi
$$

where a is the orientation of the analyzer at extinction. For $\delta 1<\pi, 2 a$ is negative and for $\delta 1>\pi, 2 a$ is positive. The problem with this method is that it is extremely difficult to obtain a plate of exact quarterwave retardance at the working wavelength. Differences up to $\pm 2^{\circ}$ from the nominal value of $90^{\circ}$ are expected in most available plates. This is clear from the two quartz quarterwave plates used in this work. The effect of deviation from exact $\lambda / 4$ value will consequently affects the retardance of the calibrated plate. Note also that the Senarmont equation Equation 6 is a special case of Equation 2,a with $p=0^{\circ}$.

We have presented a method for accurate calibration of a small retardance. The calibrated plate is a composite plate representing a full-wave phase plate. Such plate is used in some polarization experiments like photo-elasticity. As mentioned above, such plate is difficult to obtain with exact full-wave plate and is calibtated as of small retardance at $632.8 \mathrm{~nm}$. It was found that the plate is of exact full-wave retardance at $634.8 \mathrm{~nm}$ and at $632.8 \mathrm{~nm}$ its retardance is $(1.26 \pm 0.08)^{\circ}$.

\section{References}

[1] Nagib, N.N. (1999). New formulas for phase retardance measurement of birefringent plates. Opt Laser Technol., 31: 309-13.

[2] Nagib, N.N. (2004). Four-measurement technique for elimination of errors in phase plates calibration. Meas. Sci. Technol., 15: 1166-1174.

[3] Nagib, N.N., Bahrawi, M.S., Ismail, L.Z., Othman, M.H. and Abdallah, A.W. (2015). Evaluation of a photometric method for retardance measurement of a quarterwave phase plate. Opt Laser Technol. 69: 309-313.

[4] Bennett, J.M. and Bennett, H.E. (1978). In handbook of optics edited by W G Driscoll. New York: McGraw-Hill. 